Check for updates

Cite this: RSC Adv., 2017, 7, 56519

Received 30th October 2017 Accepted 11th December 2017

DOI: $10.1039 / c 7 r a 11928 c$

rsc.li/rsc-advances

\section{Synthesis of $\mathrm{pH}$-responsive nanocomposites of gold nanoparticles and graphene oxide and their applications in SERS and catalysis}

\begin{abstract}
Aihua Yao, D $\dagger^{* a b}$ Qingge Fu, $\uparrow^{\mathrm{c}}$ Ling $\mathrm{Xu},{ }^{\mathrm{b}}$ Yan $\mathrm{Xu}^{\mathrm{b}},{ }^{\mathrm{b}}$ Wenqi Jiang ${ }^{\mathrm{b}}$ and Deping Wang ${ }^{\mathrm{ab}}$
Poly(4-vinylpyridine) (P4VP), a $\mathrm{pH}$-sensitive polymer, was employed to non-covalently functionalize graphene oxides (GOs) through $\pi-\pi$ stacking interactions. Citrate-capped Au nanoparticles (Au NPs) were then immobilized onto the P4VP-grafted GO, yielding $\mathrm{pH}$-responsive Au NPs-GO nanocomposites. Such Au NPs-GO nanocomposites displayed $\mathrm{pH}$-responsive localized surface plasmon resonance (LSPR) changes and assembly behavior due to protonation and deprotonation of the pyridine units of P4VP molecules. The catalytic activity of the Au NPs-GO nanocomposites was evaluated using the reduction of 4-nitrophenol (4-NP) to 4-aminophenol (4-AP) as a model reaction, and demonstrated significantly high catalytic activity and stability at low pH (2.0), which was 4-fold higher than that at high $\mathrm{pH}$ (5.0). Furthermore, our results indicated that the Au NPs-GO nanocomposites induced active adsorption and selective SERS detection of negatively charged analytes under low pH conditions, and are therefore highly attractive for real-world chemical detection.
\end{abstract}

\section{Introduction}

Nanosized particles of noble metals, especially gold nanoparticles (Au NPs), have attracted increasing attention over the past few decades, due to their attractive electric, optical, and catalytic properties as well as potential applications in wide variety areas including physics, chemistry, biology, material science and their different interdisciplinary fields. ${ }^{1-3}$ However, $\mathrm{Au}$ NPs in solution tend to aggregate due to their high surface energy, which leads to graduate reduction in stability and catalytic activity. ${ }^{4}$ The limitation can be overcome by immobilizing $\mathrm{Au}$ NPs on diverse supports, such as $\mathrm{SiO}_{2}$, carbon, and polymer microspheres, etc. $^{5-7}$ Among these supports, graphene and its derivatives (graphene oxide, $\mathrm{GO}$, and reduced graphene oxide, RGO) have been studied extensively due to their planar structure, high surface area and excellent physicochemical properties. ${ }^{8}$ Compared to graphene, GO can be produced in a low-cost and high-yield manner, and is therefore more suitable for practical applications. Furthermore, the presence of abundant oxygen-containing functional groups on both basal planes and edges not only makes GO highly hydrophilic, but also provides a large number of active sites for surface

\footnotetext{
${ }^{a}$ Key Laboratory of Advanced Civil Engineering Materials, Ministry of Education, Tongji University, Shanghai 200092, China. E-mail: aihyao@126.com

${ }^{b}$ School of Materials Science and Engineering, Tongji University, Shanghai 200092, China

'Department of Emergency, Changhai Hospital, Second Military Medical University, Shanghai 200433, China

$\dagger$ These authors contributed equally.
}

functionalization and nanoparticle immobilization. So far, a considerable amount of research has been focused on the development of GO or RGO supported Au NPs for various applications involving catalysts, sensors, SERS, and medicine, etc. ${ }^{8-11} \mathrm{Au}$ NPs-GO nanocomposites have proven to exhibit novel and enhanced physicochemical properties, which is considered to be beneficial from a strong synergistic interaction between these two components. ${ }^{12}$

The most popular and effective strategies for synthesizing $\mathrm{Au}$ NPs-GO nanocomposites can be classified into two categories, namely, ex situ assembly and in situ growth approaches..$^{13}$ In situ growth provides better control of the size, distribution and loading density of the nanoparticles on GO sheets when compared to ex situ methods. In ex situ approaches, the modification of either GO sheets or Au NPs (or both) with suitable functional groups is a critical step for efficient assembly of $\mathrm{Au}$ NPs onto GO surfaces and improving the interfacial interaction between them. A wide range of functional groups, such as amino, pyridyl, thiol, and carboxy, etc., have been utilized to immobilize Au NPs onto GO sheets, to yield Au NPs-GO nanostructured assemblies with desired properties. ${ }^{\mathbf{8}, 14-16}$

Poly(4-vinylpyridine) (P4VP) is an attractive polymer and has been widely used as a surface modifier because of the pyridyl group's strong affinity to transition metals. ${ }^{17,18}$ Additionally, $\mathrm{P} 4 \mathrm{VP}$ is well-known as a pH-responsive polymer that undergoes molecular conformational changes at a critical transition $\mathrm{pH}$ value $(\sim 4.5) .{ }^{19}$ At low $\mathrm{pH}(<4.5)$, the pyridyl segment of P4VP is protonated by reactions with acids and forms positively charged pyridinium groups, which results in the chain stretching under the electrostatic repulsion. Conversely, the pyridine exists in 
a deprotonated form at higher $\mathrm{pH}$ ranges $(>4.5)$, and the polymer chains adopted a coil conformation. Such a $\mathrm{pH}$ effect of P4VP has been used to functionalize GO and Au NPs for various applications. For example, pH responsive Au@P4VP nanocomposites were developed utilizing Au NPs or nanorods decorated with P4VP molecules. It was found that the resulting nanocomposites exhibited pH-dependent localized surface plasmon resonance (LSPR) due to the conformational transition of P4VP in different $\mathrm{pH}$ environments, and therefore LSPRbased sensing or catalytic applications were explored. ${ }^{20-22}$ P4VP-grafted GO displayed reversible electrochemical response to $\mathrm{pH}$ changes. The protonation/deprotonation of pyridine units induced significant changes in the microenvironment of GO/P4VP assembles, and allowed for applications in $\mathrm{pH}$ induced switchable bioelectronics, intelligent nanodevices and pH-triggered drug delivery system. ${ }^{\mathbf{1 1 , 2 3 , 2 4}}$ Recently, Li et al. ${ }^{6}$ synthesized core-shell C@P4VP microspheres by coating P4VP shells on the surfaces of carbon spheres, and then the microspheres were used to support Au NPs for catalytic reduction of 4nitrophenol. Their results indicated that the polymeric P4VP shell not only endowed the catalyst with smart $\mathrm{pH}$ responsibility but also enhanced its catalytic activity and cycle stability.

Inspired by the studies above, we developed Au NPs-GO nanocomposites based on P4VP-grafted GO nanosheets decorated with $\mathrm{Au}$ NPs. It was expected that the conformational transition of P4VP chains would result in a tunable interfacial interaction between GO and Au NPs, and allowed for controlled performance. Our results indicated that the presence of the P4VP not only functionalized the GO surfaces, enabling the immobilization of Au NPs with high and uniform distribution on GO sheets, but also endowed the nanocomposites with $\mathrm{pH}$ responsive behavior. We therefore explored their potential applications in SERS and catalysis, and found that the Au NPsGO nanocomposites displayed $\mathrm{pH}$-dependent catalytic activity towards the reduction of 4-NP, and selective SERS detection of negatively-charged dye molecules.

\section{Experimental}

\section{Chemicals}

Graphite flakes (nature, -10 mesh) were purchased from Alfa Aesar (Tianjin, China). $\mathrm{HAuCl}_{4} \cdot 4 \mathrm{H}_{2} \mathrm{O}$ and sodium citrate $\left(\mathrm{C}_{6}\right.$ $\mathrm{H}_{5} \mathrm{Na}_{3} \mathrm{O}_{7} \cdot 2 \mathrm{H}_{2} \mathrm{O}$ ) were purchased from Shanghai Aladdin Chemical Reagent Company. Dimethyl sulfoxide (DMSO), and poly(4-vinylpyridine) ( $\left.\mathrm{P} 4 \mathrm{VP}, M_{\mathrm{w}}=60000\right)$ were purchased from Sigma-Aldrich. All reagents were analytical grade and used without further purification. All aqueous solutions were prepared with twice-distilled water $(18.2 \mathrm{M} \Omega \mathrm{cm})$.

\section{Synthesis of Au NPs-GO nanocomposites}

Preparation and modification of GO. GO was synthesized as reported previously. ${ }^{25}$ P4VP-modified GO was prepared as follows: $20 \mathrm{mg}$ of P4VP was first dissolved in $25 \mathrm{~mL}$ of DMSO, and then $10 \mathrm{mg}$ GO was added to the solution. After 2 hours of ultrasonication, the resulting mixture was centrifuged at
$10000 \mathrm{rpm}$ and washed with diluted $\mathrm{HCl}$ solution $(\mathrm{pH} 2.0)$ atleast three times to remove excess P4VP. The final P4VPmodified GO was redispersed in DMSO and stored; the final concentration of GO was about $2.5 \mathrm{mg} \mathrm{mL}^{-1}$.

Synthesis of Au NPs-GO nanocomposites. Au NPs sol was synthesized by adding $3 \mathrm{~mL}$ of sodium citrate solution (38 $\mathrm{mmol}$ ) to $50 \mathrm{~mL}$ of boiling $\mathrm{HAuCl}_{4} \cdot 4 \mathrm{H}_{2} \mathrm{O}$ aqueous solution (1.4 $\mathrm{mmol}$ ). The solution was continuously stirred and boiled for $10 \mathrm{~min}$. Subsequently, $25 \mathrm{~mL}$ of GO/DMSO suspension was added to the resulting $\mathrm{Au}$ sol, and the mixture was ultrasonicated for $3 \mathrm{~h}$. The final product was centrifuged at $10000 \mathrm{rpm}$ and washed with deionized water three times and dried under vacuum at $60{ }^{\circ} \mathrm{C}$.

pH responsibility of Au NPs-GO nanocomposites. The $\mathrm{Au}$ NPs-GO nanocomposites were dispersed in $\mathrm{HCl}$ solutions of $\mathrm{pH}$ 2.0-5.0, DI water and $\mathrm{NaOH}$ solution of $\mathrm{pH} 10.0$, respectively. Subsequently these dispersions were ultrasonicated and incubated at room temperature for $5 \mathrm{~h}$ to reach adsorption equilibrium. The zeta potential of the materials was then determined, and UV-vis spectra were recorded from 300 to $700 \mathrm{~nm}$. Furthermore, the Au NPs-GO nanocomposites were dispersed in $\mathrm{HCl}$ solutions of $\mathrm{pH} 2.0$ and 5.0, and were dropped onto carbon-coated copper grid for TEM observation.

Catalytic reduction of 4-NP. Catalytic reduction of 4-NP by $\mathrm{NaBH}_{4}$ was chosen as a model reaction to evaluate the catalytic activity of the resulting Au NPs-GO nanocomposites. Au NPsGO nanoparticles dispersed in $\mathrm{HCl}$ solutions of $\mathrm{pH} 2.0$ and 5.0 $\left(0.1 \mathrm{~mL}, 0.2 \mathrm{mg} \mathrm{mL}^{-1}\right)$ were mixed with $0.2 \mathrm{~mL}$ of $4-\mathrm{NP}$ aqueous solution $(10 \mathrm{mM})$ in a quartz cell. $1 \mathrm{~mL}$ of fresh $\mathrm{NaBH}_{4}$ aqueous solution $(0.2 \mathrm{M})$ was then added to the mixture under stirring. The reduction reaction was monitored by UV-vis spectroscopy by measuring the intensity of the $4-\mathrm{NP}$ peak at $400 \mathrm{~nm}$ at given time intervals. For the reusable test, the catalyst was collected, washed with deionized water and dried for the next cycle.

Preparation of SERS samples. $3.4 \mathrm{~mL}$ of aqueous solutions of rhodamine 6G (R6G), methylene blue (MB), trypan blue (TB) and Ponceau S (PS) $\left(2.5 \times 10^{-3} \mathrm{M}\right)$ were dropped into Au NPsGO dispersions at $\mathrm{pH} 2.0$ and $5.0\left(600 \mu \mathrm{L}, 0.2 \mathrm{mg} \mathrm{mL}^{-1}\right)$, respectively. The solutions were then ultrasonicated for $30 \mathrm{~min}$ at room temperature for sufficient adsorption. Subsequently 30 $\mu \mathrm{L}$ of the solution was dropped onto the glass slides and air dried at ambient conditions for SERS measurement.

Characterization. The morphology and structure of the Au NPs-GO nanocomposites were characterized using a transmission electron microscope (TEM, Hitachi S-2360) with an accelerating voltage of $200 \mathrm{kV}$. X-ray powder diffraction (XRD) was conducted on a Rigaku D max 2550 diffractometer using $\mathrm{Cu}$ $\mathrm{K} \alpha$ radiation $(\lambda=1.5418 \AA)$. UV-vis detection was recorded on a LAMBDA 750 spectrometer (PerkinElmer). Thermogravimetric analysis (TGA) was conducted on Q600-SDT simultaneous TGADSC in air at a heating rate of $10{ }^{\circ} \mathrm{C} \min ^{-1}$. The elemental composition was determined by an X-ray photoelectron spectroscopy (XPS, Escalab250Xi, Thermo Scientific) using a $500 \mu \mathrm{m}$ diameter beam of monochromatic $\mathrm{Al} \mathrm{K} \alpha$ radiation. The zeta potential of the materials was determined from Dynamic Light Scattering system (Zeta SIZER NANO ZS90, MALVERN). SERS measurements were conducted with a Renishaw inVia micro- 
Raman spectrometer with He-Ne laser excitation at $532 \mathrm{~nm}$. A $100 \times$ objective was used to focus the laser beam and to collect the Raman signals. The SERS spectra were collected from at least 5 random locations with an accumulation time of $10 \mathrm{~s}$, and expressed in terms of average spectra.

\section{Results and discussion}

\section{Synthesis and characterization of Au NPs-GO nanocomposites}

Initial GO suspensions were prepared according to the modified Hummers method from commercial graphite. Surface grafting was carried out using P4VP molecules with the aid of sonication. Further interactions between P4VP-GO conjugates and citrate-capped $\mathrm{Au}$ NPs resulted in Au NPs-GO nanocomposites. A successful decoration of Au NPs on GO sheets was verified by TEM observation. As shown in Fig. 1(a), the GO sample is composed of few-layer sheets and exhibits typical wrinkled morphology. After the assembly process, the GO sheets were decorated with well-dispersed spherical Au NPs (Fig. 1(b) and (c)). The mean size of Au NPs anchored on the GO was about $20 \mathrm{~nm}$ as determined by measuring diameters of total 200 particles from 5 TEM images (the inset of (c)). It is noteworthy that there are almost no free Au NPs outside the domain of the GO sheets, indicating a strong interaction between the Au NPs and P4VP-grafted GO. The high-resolution TEM image in Fig. 1(d) reveals that the Au NPs have a highly crystalline structure with an interplane distance of $\sim 0.235 \mathrm{~nm}$ that corresponds to the spacing of (111) plane of a face-centered cubic (fcc) Au crystal.
XRD pattern in Fig. 2(a) further verifies the fcc structure of Au NPs (JCPDS no. 04-0784). The average size of Au NPs was determined to be $21.2 \mathrm{~nm}$ according to the Scherrer formula, consistent with the size observed by TEM images. Moreover, a weak diffraction peak around $2 \theta=25^{\circ}$ corresponding to the (002) plane of GO is also detected. The elemental composition and interfacial interaction between GO and Au NPs were checked by XPS technique. The survey scan profile in Fig. 2(b) confirms that the Au NPs-GO nanocomposites contain C, O, Au, and $\mathrm{N}$ elements. The weak $\mathrm{N}$ signal originates from the pyridine moieties of P4VP chain, indicating the existence of P4VP molecules on GO surfaces. The deconvolution of the $\mathrm{C} 1 \mathrm{~s}$ spectrum resulted in four different components. As shown in Fig. 2(c), the predominant peak at $284.6 \mathrm{eV}$ corresponds to nonoxygenated carbon bonds in both GO and P4VP. The peak at $286.4 \mathrm{eV}$ is attributed to the $\mathrm{C}-\mathrm{O}$ bonds in $\mathrm{GO}$ and $\mathrm{C}-\mathrm{N}$ bonds in $\mathrm{P} 4 \mathrm{VP}$. The broad peak at $287.8 \mathrm{eV}$ corresponds to $\mathrm{C}=\mathrm{O}$ bonds in GO. The C 1s spectrum further shows a small signal at $291.7 \mathrm{eV}$, which is due to a $\pi-\pi^{*}$ shake-up signal characteristic of aromatic $\pi$ electrons. This signal has been observed for similar polymer-GO composites. ${ }^{26,27}$ These results indicated that the GO sheets have been non-covalently functionalized with P4VP molecules through $\pi-\pi$ interactions between the delocalized $\mathrm{sp}^{2}$ carbon structure of GO and the pyridine moieties of P4VP chains. The Au $4 \mathrm{f}$ core-level spectrum in Fig. 2(d) reveals Au $4 \mathrm{f}$ doublet peaks at 83.8 and $87.5 \mathrm{eV}$. These peaks are shifted to lower binding energies when compared to the standard XPS spectrum of metallic $\mathrm{Au}^{0}$ (84.0 and $87.7 \mathrm{eV}$ ), indicating the electron transfer from GO to Au NPs. ${ }^{28}$

Fig. 3(a) shows UV-vis absorption spectra of P4VP-grafted GO (P4VP/GO) and Au NPs-GO nanocomposites dispersed in DMSO
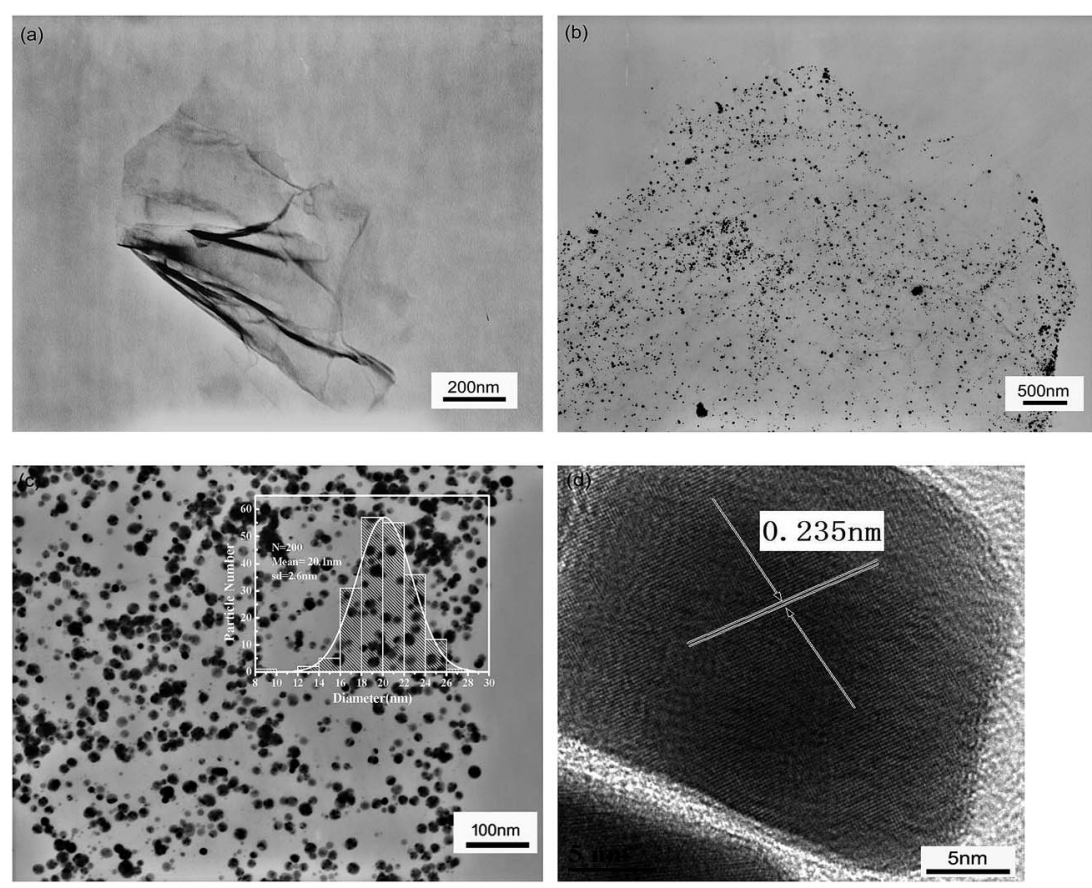

Fig. 1 (a-c) TEM images of GO sheets and Au NPs-GO nanocomposites with different magnifications; (c) HRTEM image of a representative Au NPs-GO nanoparticle. 

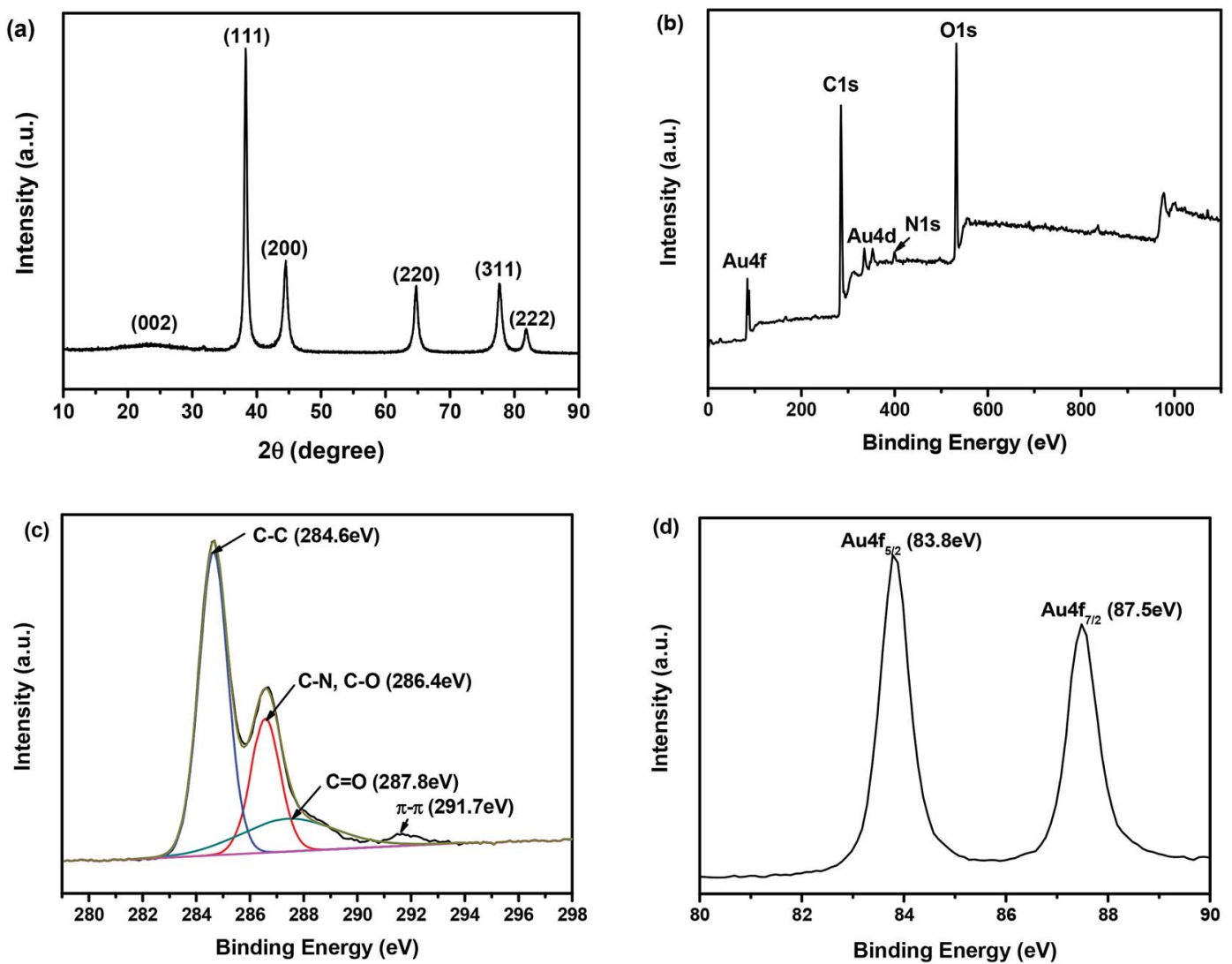

Fig. 2 (a) XRD pattern of Au NPs-GO nanocomposites; (b-d) XPS survey scan, C 1s deconvolution and Au 4f analysis of Au NPs-GO nanocomposites.

and are compared with neat GO and P4VP. The spectrum of GO (curve II) exhibits an absorption peak at $234 \mathrm{~nm}$ and a shoulder peak at $300 \mathrm{~nm}$, which can be assigned to the $\pi-\pi *$ transition of $\mathrm{C}=\mathrm{C}$ bonds and $\mathrm{n}-\pi^{*}$ transition of $\mathrm{C}=\mathrm{O}$ bonds, respectively. ${ }^{29}$ A strong absorption peak at $287 \mathrm{~nm}$ arising from P4VP polymer is observed in the spectrum of $\mathrm{P} 4 \mathrm{VP} / \mathrm{GO}$ (curve III), which provides further evidence for the successful surface grafting of GO by the P4VP molecules. Further decoration of Au NPs on $\mathrm{P} 4 \mathrm{VP} / \mathrm{GO}$ results in a new absorption peak at $\sim 530 \mathrm{~nm}$ that corresponds to the LSPR of Au NPs (curve IV).

The mass percentage of $\mathrm{Au}$ in the $\mathrm{Au}$ NPs-GO nanocomposites was analysed via TGA. In Fig. 3(b), the small weight loss below $150{ }^{\circ} \mathrm{C}$ is attributed to loss of adsorbed water and residual DMSO. The weight loss of $10 \%$ between 200 and $500{ }^{\circ} \mathrm{C}$ is associated with the thermal degradation of P4VP molecules, citrate capping on the Au NPs, and some oxygen-containing functional groups of GO. Upon increasing the temperature up to $800{ }^{\circ} \mathrm{C}$, a $50 \%$ weight loss is observed and is attributed to decomposition of the carbon skeleton of GO. Accordingly, the mass percentage of Au NPs is determined to be around 36\% of the total nanocomposites based on the remaining weight at the end of the experiment.

Pyridine, with an aromatic structure, has a strong tendency to interact with GO sheets via $\pi-\pi$ stacking. Simultaneously
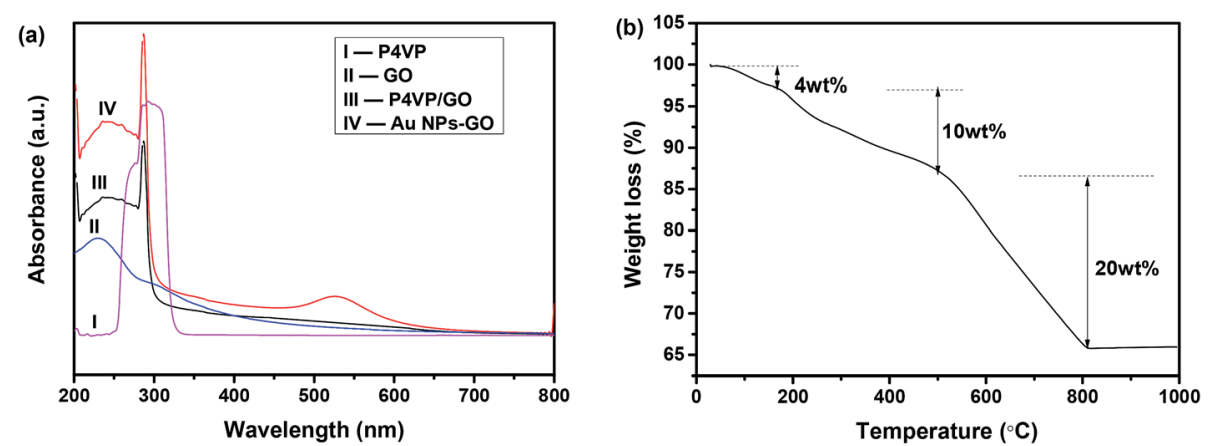

Fig. 3 (a) UV-vis absorption spectra of P4VP-grafted GO and Au NPs-GO nanocomposites dispersed in DMSO, GO and P4VP; (b) TGA curve of Au NPs-GO nanocomposites. 

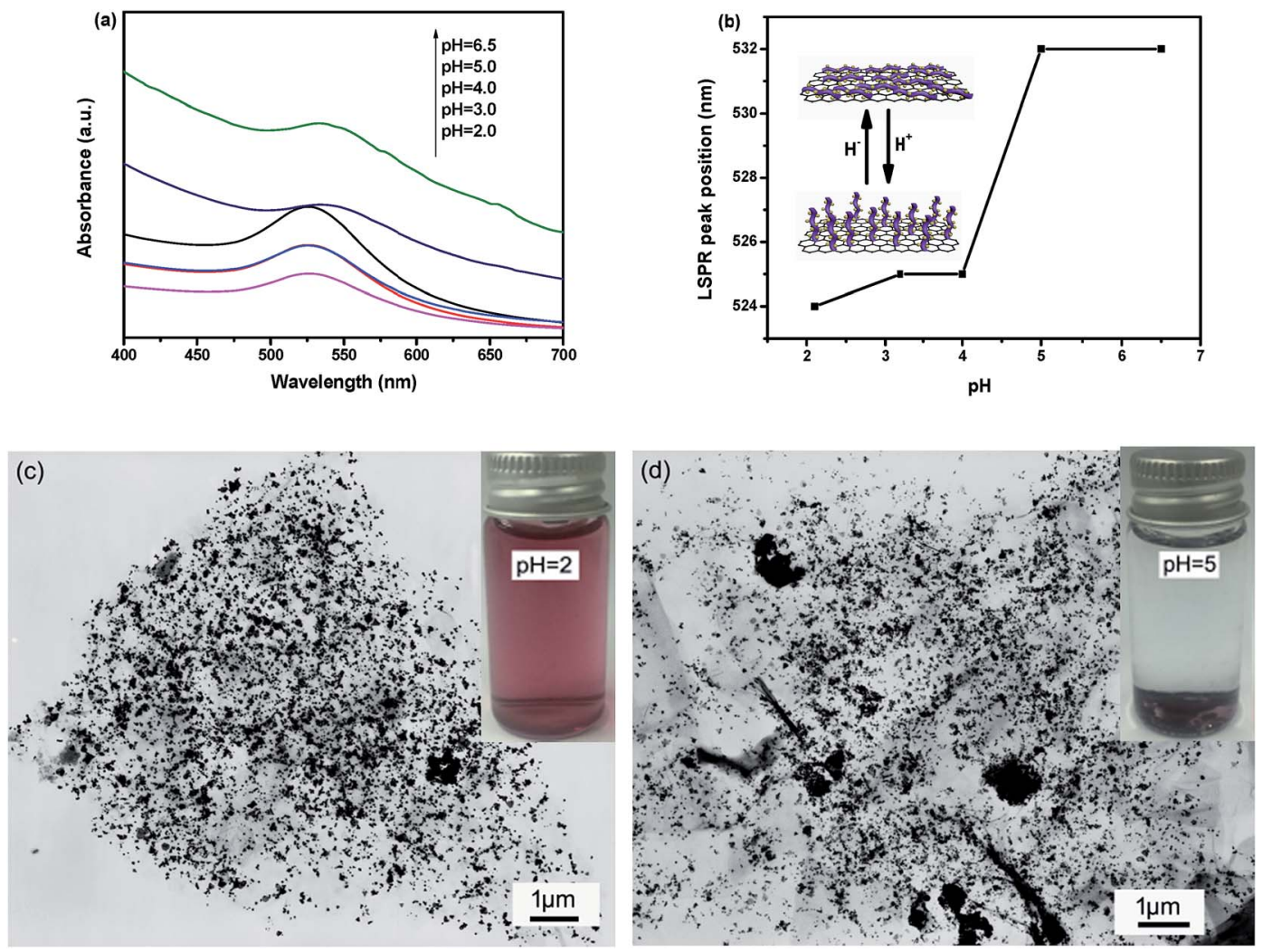

Fig. 4 (a) UV-vis spectra of the Au NPs-GO nanocomposites dispersed in dilute HCl solution of pH 2.0-5.0 and DI water (pH 6.5); (b) LSPR peak positions changes of the Au NPs-GO nanocomposites at various $\mathrm{pH}$; the inset of (b) shows scheme of $\mathrm{pH}$-responsive morphology change of the Au NPs-GO nanocomposites. (c and d) TEM images of the Au NPs-GO nanocomposites at pH 2.0 and pH 5.0; the insets of (c) and (d) show photographs of the Au NPs-GO nanocomposites at $\mathrm{pH} 2.0$ and $\mathrm{pH} 5.0$

pyridine groups have good binding affinity to Au NPs via the formation of hydrogen bonding with the citrate capping on the surfaces of $\mathrm{Au}$ NPs. Therefore, after the formation of $\pi-\pi$ stacking interactions with GO, P4VP still contains a large number of unbound pyridyl groups that are capable of binding to Au NPs. As a result of the free pyridines, the Au NPs are well anchored to the GO sheets with high coverage density, and therefore providing sufficient amount of catalytic active sites and highly efficient electron transfer between the Au NPs and GO, which may subsequently enhance the catalytic activity and SERS effect of the nanocomposites.

\section{pH response of Au NPs-GO nanocomposites}

To evaluate the $\mathrm{pH}$ response of Au NPs-GO nanocomposites, the samples were dispersed in dilute $\mathrm{HCl}$ solution of $\mathrm{pH}$ 2.0-5.0 and deionized water ( $\mathrm{pH}$ 6.5), respectively. After equilibrium adsorption, the UV-vis absorption spectra of all samples were recorded from 400 to $700 \mathrm{~nm}$. Fig. 4(a) clearly displays that the LSPR peaks of Au NPs change from $524 \mathrm{~nm}$ at pH 2.0 to $532 \mathrm{~nm}$ at $\mathrm{pH}$ 6.5, with around $8 \mathrm{~nm}$ red-shift. Simultaneously, broadening of the absorption peak occurs with an increase in $\mathrm{pH}$. The LSPR peak positions of Au NPs were plotted $v s$. $\mathrm{pH}$ values and presented in Fig. 4(b). With increasing $\mathrm{pH}$, the LSPR peak shows small changes at $\mathrm{pH}<4.0$, but a dramatic increase as $\mathrm{pH}$ changed from 4.0 to 5.0 , and eventually reaches a nearly constant wavelength of $\sim 532 \mathrm{~nm}$. Such pH-dependent LSPR absorption of the Au NPs-GO is similar to those observed in P4VP-grafted Au nanoparticles and nanorods..$^{20,21}$

The pH-responsive behavior of the Au NPs-GO nanocomposites was further evaluated through zeta potential analysis and TEM observation. The insets in Fig. 4(c) and (d) show the photographs of the nanocomposites dispersed in dilute $\mathrm{HCl}$ solutions at $\mathrm{pH} 2.0$ and 5.0. It was found that the dispersion of $\mathrm{Au}$ NPs-GO nanocomposites was dependent on the $\mathrm{pH}$ value of the medium. At $\mathrm{pH}=2.0$, the nanocomposites were well dispersed in aqueous solution, and formed a reddish black homogeneous dispersion. However, obvious aggregation occurred and resulted in black precipitates at the bottom of the vessel when the $\mathrm{pH}$ was changed to 5.0. If the medium $\mathrm{pH}$ was adjusted back to 2.0 , the precipitates were able to be redispersed into the solution again, confirming a reversible $\mathrm{pH}^{-}$ responsive behavior. The zeta potential measurements revealed that the particle zeta potential maintained a high value of $\sim 32.5 \mathrm{mV}$ at $\mathrm{pH} 2.0$, and such a high surface charge prevented the aggregation of the nanocomposites, thus allowing good dispersion. Conversely, at a higher $\mathrm{pH}$ (5.0), the zeta potential drastically decreased to $5.7 \mathrm{mV}$, and nearly went to zero under neutral conditions. Upon further increasing $\mathrm{pH}$ to 10.0 , the zeta potential value was $-7.2 \mathrm{eV}$. The negative zeta potential of the nanocomposites originated from the 

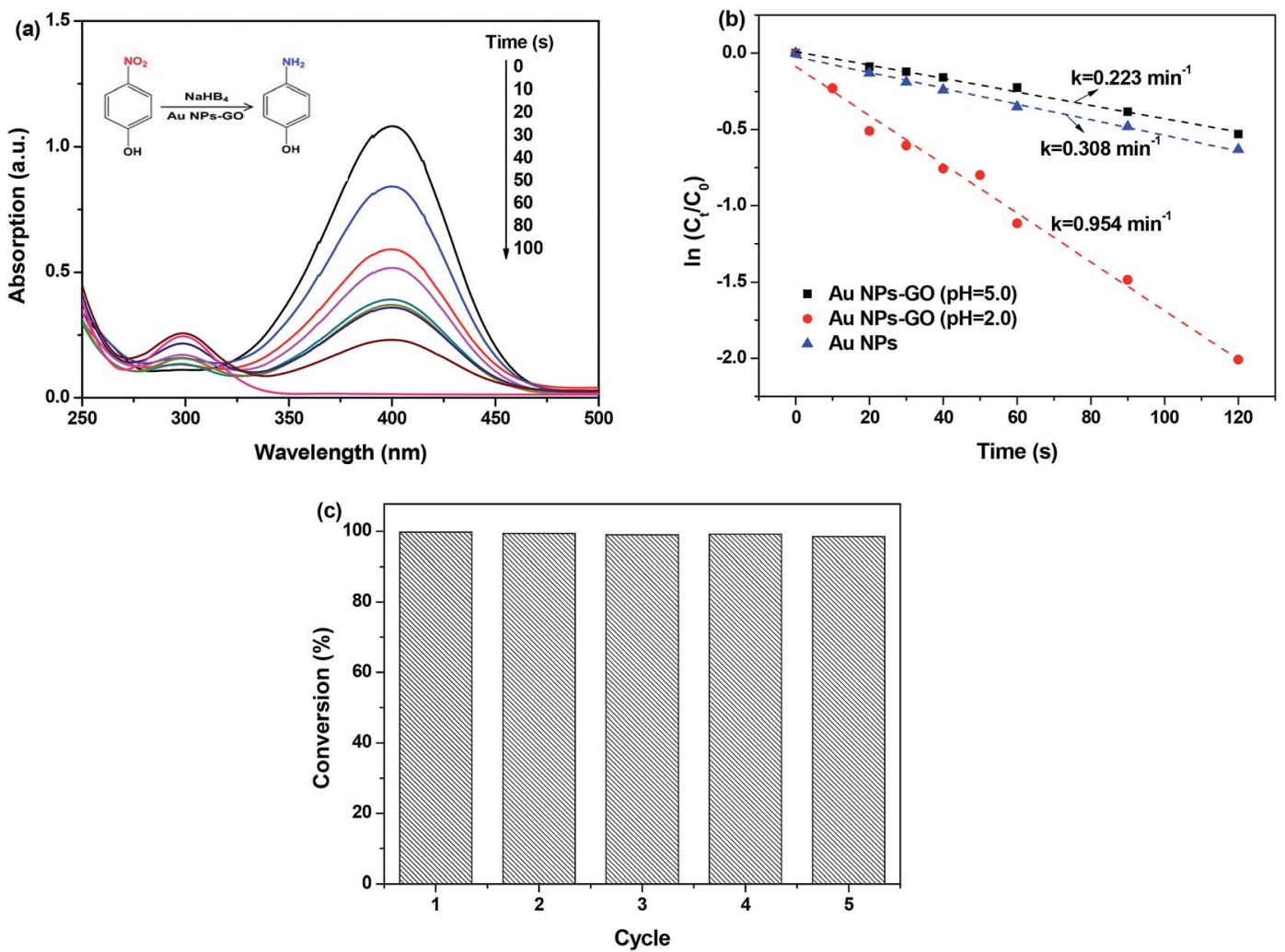

Fig. 5 UV-vis spectra of the reduction reaction of 4-NP to 4-AP with Au NPs-GO nanocomposites at different time intervals; $(b)$ the $\ln \left(C_{t} / C_{0}\right)$ vs. $t$ plots for the reduction reaction of 4-NP in the presence of Au NPs-GO nanocomposites at pH 2.0 and 5.0 and Au NPs. Conditions: [4-NP] $=$ $10 \mathrm{mM}$; [Au NPs-GO catalysts] $=0.2 \mathrm{mg} \mathrm{mL}^{-1}$; [Au NPs catalysts] $=0.08 \mathrm{mg} \mathrm{mL}^{-1}$; $\left[\mathrm{NaBH}_{4}\right]=0.2 \mathrm{M}$; (c) reusability test of Au NPs-GO nanocomposites as catalysts for the reduction of 4-NP.

deprotonation of citrate anions capping on Au NPs as well as the carboxylic acid and phenolic hydroxyl groups on GO sheets. Nevertheless, the zeta potential values of the nanocomposites at $\mathrm{pH}>5.0$ are not enough to achieve good particle suspension stability. TEM images in Fig. 4(c) and (d) directly reveal the $\mathrm{pH}^{-}$ response of the Au NPs-GO nanocomposites. Large agglomerates of Au NPs are obviously formed on the GO sheets as the $\mathrm{pH}$ value of the media is changed from 2.0 to 5.0.

Such a pH-responsive behavior of the Au NPs-GO nanocomposites is ascribed to the protonation/deprotonation of pyridine groups on the grafted P4VP. At low pH $(<4.0)$, the pyridyl groups are protonated to form pyridinium ions, and renders the Au NPs-GO nanocomposites with positive charges, as evidenced by the high zeta potential. Meanwhile the positively charged polymer chains repulse each other, and result in an increased distance between the Au NPs. The increased distance between $\mathrm{Au}$ NPs is verified by the observed good dispersion of $\mathrm{Au}$ NPs on $\mathrm{GO}$ sheets at $\mathrm{pH} 2.0$ versus poor dispersion at $\mathrm{pH} 5.0$ due to a lack of electrostatic repulsion. Thus, large Au NPs agglomerations are observed on GO sheets at $\mathrm{pH} 5.0$ as shown in TEM, accompanied by the redshift and broadening of UV-vis absorption peaks. However, the electrostatic repulsion between negatively charged citrate capped-Au NPs limited agglomeration to a certain degree, so that the SPR peaks shifts to a constant wavelength of about $532 \mathrm{~nm}$. On the other hand, the P4VP chains collapse onto the GO surfaces at high $\mathrm{pH}$, and increase the refractive index around Au NPs, which can also result in a red shift of Au LSPR peaks. ${ }^{30}$

\section{pH-responsive catalysis activity of the Au NPs-GO nanocomposites}

The catalytic activity of the Au NPs-GO nanocomposites was evaluated using the reduction of 4-NP to 4-AP as a model reaction. It has been reported that Au NPs act as an effective catalyst for the reduction of 4-NP in the presence of $\mathrm{NaBH}_{4}$ by facilitating electron transfer from $\mathrm{BH}_{4}{ }^{-}$to $4-\mathrm{NP}^{31,32}$ To compare the catalytic performance of the nanocomposites at various $\mathrm{pH}$ levels, the Au NPs-GO nanocomposites were initially dispersed in dilute $\mathrm{HCl}$ solution of $\mathrm{pH} 2.0$ and 5.0. These solutions were subsequently injected into a mixture of 4-NP and $\mathrm{NaBH}_{4}$. It was found that the dark yellow color of mixture solution was quickly quenched. The fast reaction rate is evidenced by the sharp decrease of the absorption peaks at $400 \mathrm{~nm}$ in UV-vis spectra (Fig. 5(a)). Meanwhile, new peaks corresponding to 4-AP appeared at $300 \mathrm{~nm}$ appear and increase over time. As shown in Fig. 5(a), the reduction of 4-NP to 4-AP is finished within $100 \mathrm{~s}$ using Au NPs-GO nanocomposite catalysts at $\mathrm{pH}$ 2.0. Pseudofirst order kinetics was utilized to determine the reaction rate constant $(k)$, which can be described by the equation:

$$
\ln \left(\frac{C_{t}}{C_{0}}\right)=-k t
$$



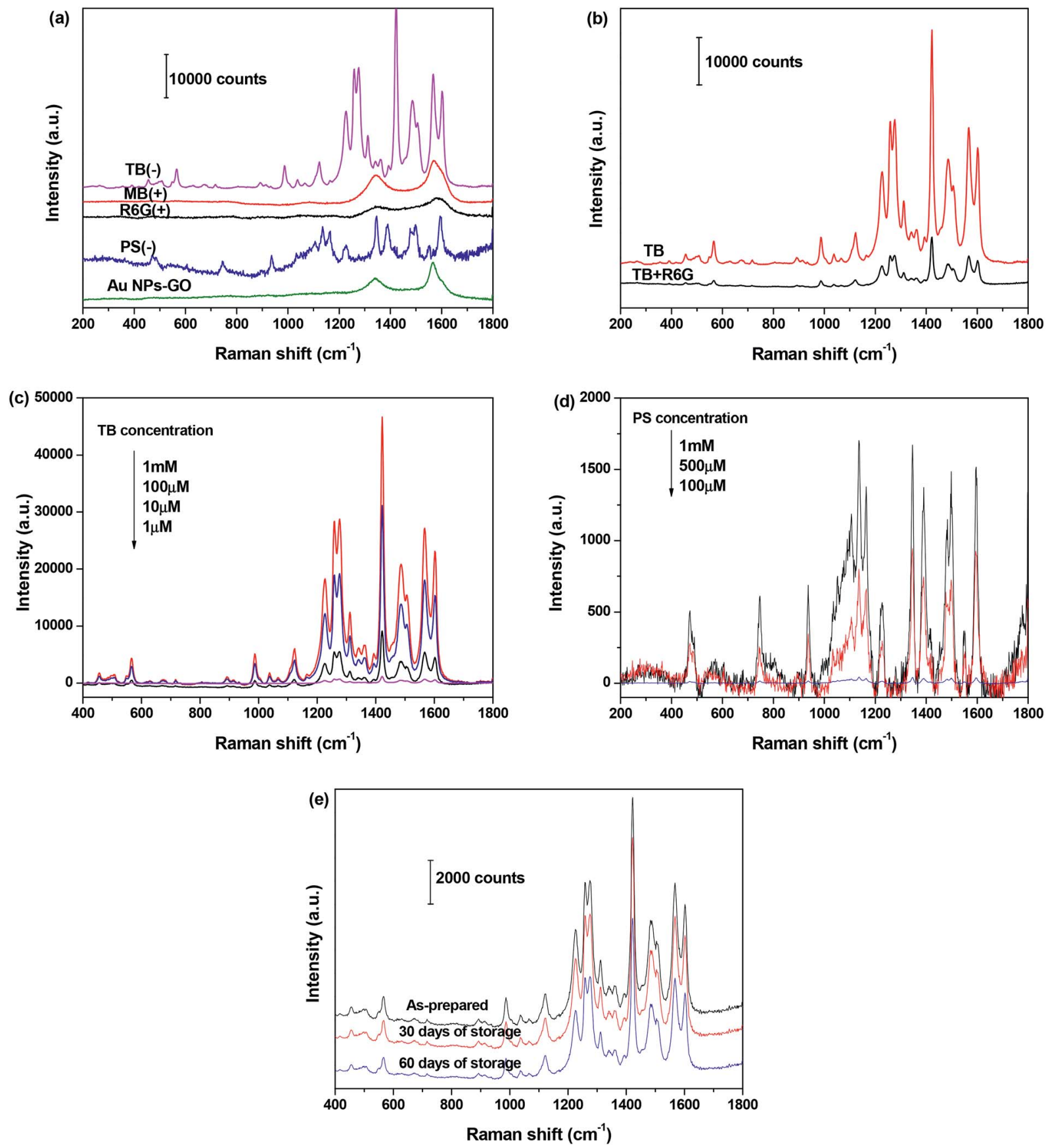

Fig. 6 SERS spectra of $1 \mathrm{mM}$ aqueous solutions of R6G (+), MB (+), TB (-) and PS (-) adsorbed on the Au NPs-GO nanocomposites at pH 2.0; (b) SERS spectra of $1 \mathrm{mM}$ TB and dye mixture containing R6G and TB (1 mM each) on the Au NPs-GO nanocomposites; (c and d) SERS spectra of different concentrations of TB and PS adsorbed on Au NPs-GO nanocomposites; (e) SERS spectra of TB (10 $\mu$ M) absorbed on Au NPs-GO after storage in the refrigerator for 0,30 , or 60 days.

where $C_{0}$ and $C_{t}$ represent the 4 -NP concentrations at time 0 and time $t$, respectively. Fig. 5(b) shows a well-defined linear relationship between $\ln \left(C_{t} / C_{0}\right)$ and time, and the corresponding rate constants are determined from the slopes of the plotted lines. As observed, the reaction rate constant $(k)$ is raised significantly from 0.223 to $0.954 \mathrm{~min}^{-1}$ when the $\mathrm{pH}$ values of the reaction change from 5.0 to 2.0. For comparison purpose, the same amount of citrate-capped Au NPs as those anchored on the GO sheets were also evaluated under the same conditions and the $k$ value was calculated to be $0.308 \mathrm{~min}^{-1}$. These comparative results demonstrate the superior catalytic capability of the Au NPs-GO catalyst at $\mathrm{pH}=2.0$. Furthermore, it is noteworthy that the present Au NPs-GO nanocomposites at low $\mathrm{pH}$ value exhibit a higher catalytic activity than recently reported Au-based catalysts, ${ }^{26,33}$ and are even comparable to Ptbased catalysts for the reduction of 4-NP. ${ }^{26}$ 
For the Au NPs-GO nancomposites incubated in the $\mathrm{HCl}$ solution at $\mathrm{pH} 2$, the protonated polymer chains of P4VP tend to extend maximally under the electrostatic repulsion. In this case, the Au NPs bound to the P4VP can easily access the reagents (4$\mathrm{NP}$ and $\mathrm{HB}_{4}{ }^{-}$), which increase the number of accessible catalytic active sites. Further, the extended chain morphology facilitate the electron transfer between the GO and Au NPs, increasing local electron concentration. The higher electron concentration favors the uptake of electrons by 4-NP molecules. ${ }^{34}$ Meanwhile, $\mathrm{BH}_{4}{ }^{-}$ions are readily to absorbed on the positively charged Au NPs-GO nanocomposites and relayed electrons to the active sizes for reduction of $4-\mathrm{NP} .{ }^{35}$ However in the case of $\mathrm{pH}$ 5.0, the P4VP chains adopted a coiled conformation, resulting in the inclusion of Au NPs within the polymer chains and thus partial catalytic active sites were shielded. The reduction of catalytic active sites resulted in relatively low catalytic activity.

Since catalytic stability is highly desirable for catalyst in practical applications, the reusability of the Au NPs-GO nanocomposites was evaluated by successively testing their catalytic performance. For this purpose, the catalyst was collected, washed and redispersed in dilute $\mathrm{HCl}$ solution of $\mathrm{pH} 2.0$ for the next cycle. The conversion of 4-NP to 4-AP was calculated as $1-$ $A_{t} / A_{0}$, where $A_{t}$ represents the absorption of $4-\mathrm{NP}$ at $400 \mathrm{~nm}$ at $100 \mathrm{~s}$, and $A_{0}$ is the absorption of 4-NP in original reactant. From Fig. 5(c), no measured decease in the catalytic activity was observed after running for 5 successive cycles, demonstrating the excellent catalytic stability of the Au NPs-GO nanocomposites.

\section{SERS activity of the Au NPs-GO nancomposites}

Inspired by the high zeta potential of the Au NPs-GO nanocomposites at $\mathrm{pH}<4.0$, we hypothesized that the negatively charged analyte molecules would preferentially adsorb onto sample surfaces, and subsequently the anchored Au NPs enhanced their Raman signals; whereas the adsorption of positively charged analytes would be unfavorable due to electrostatic repulsion, and therefore enabled the charge-selective SERS analysis of dye molecules. Herein two positively charged analytes, rhodamine 6G (R6G) and methylene blue (MB), and two negatively charged analytes, trypan blue (TB) and ponceau $S$ (PS), were employed as representative Raman probes to demonstrate the hypothesis. The Au NPs-GO nanocomposites that were well-dispersed in the $\mathrm{HCl}$ solution of $\mathrm{pH} 2.0$ were mixed absolutely with the aqueous solution of each analyte, and then the mixture solution was dropped onto glass slides for SERS measurement.

Fig. 6(a) compares the SERS spectra of $1 \mathrm{mM}$ aqueous solution of R6G, MB, TB and PS recorded under the same conditions. Distinct Raman bands corresponding to TB and PS molecules were observed, suggesting a strong SERS enhancement for the negatively charged analyte molecules. Conversely, the SERS spectra collected from R6G and MB molecules are similar to that of the Au NPs-GO nanocomposites. Only typical $\mathrm{D}$ and $\mathrm{G}$ bands of the GO component at 1345 and $1580 \mathrm{~cm}^{-1}$ were observed, but no characteristic Raman bands of the analyte molecules were detected. The charge selectivity was further tested using a mixed solution of TB and R6G (concentration of $1 \mathrm{mM}$ each). The SERS spectrum in Fig. 6(b) demonstrates a decrease in intensity of characteristic TB Raman signals compared with single TB solution, yet is still clearly distinguishable. These results clearly indicate the high sensitivity and selectivity of SERS detection for negatively charged analytes with the Au NPs-GO nanocomposites under low $\mathrm{pH}$ conditions.

We further measured the SERS spectra as a function of the dye concentration and determined the detection sensitivity. As expected, the Raman signal intensities of TB and PS decreases with decreasing concentrations (Fig. 6(c) and (d)). For TB, the Raman band is clearly distinguishable down to $1 \mu \mathrm{M}$ concentration, which was 100 times lower than that of PS. The difference in detection sensitivity between the two analysts is attributed to a stronger affinity between TB and substrate than PS and the substrate.

The stability of Au NPs-GO nanocomposites was assessed by determining the Raman peak intensity of adsorbed TB $(10 \mu \mathrm{M})$ after storage in the refrigerator for 0,30 , or 60 days. The spectra in Fig. 6(e) presents that the Raman signal intensity of TB decreases to approximately $8 \%$ and $30 \%$ of its initial value after 30 and 60 days of storage, respectively. These results indicate that the Au NPs-GO nanocomposites are stable as a substrate and produce reliable SERS measurements.

\section{Conclusions}

The Au NPs-GO nanocomposites were successfully synthesized by decorating Au NPs onto P4VP-grafted GO sheets. The presence of P4VP polymer not only enabled the immobilization of $\mathrm{Au}$ NPs with high and uniform distribution on GO sheets, but also endowed the nanocomposites with $\mathrm{pH}$-sensitive characteristics. We demonstrated that the as-prepared Au NPs-GO nanocomposites displayed $\mathrm{pH}$-responsive LSPR changes and assembly behavior. The Au NPs-GO nanocomposites were employed as efficient catalysts for the reduction of 4-NP, and demonstrated $\mathrm{pH}$-dependent catalytic activity. Further investigation indicated that the nanocomposites exhibited selective adsorption and high-efficient SERS detection of negativelycharged dye molecules. Therefore, the Au NPs-GO nanocomposites provide a new opportunity for real-world chemical detection and catalysis.

\section{Conflicts of interest}

There are no conflicts to declare.

\section{Acknowledgements}

This work was financially supported by National Natural Science Foundation of China (No. 50702037), Natural Science Foundation of Shanghai Municipality (No. 16ZR1400700), Shanghai Health and Family Planning Commission Project (No. 2012y193) and Research Funds for the Central Universities. 


\section{References}

1 E. Priyadarshini and N. Pradhan, Sens. Actuators, B, 2017, 238, 888-902.

2 W. Zhou, X. Gao and D. B. Liu, Chem. Rev., 2015, 115, 1057510636.

3 L. Wang, H. L. Li, J. Q. Tian and X. P. Sun, ACS Appl. Mater. Interfaces, 2010, 11, 2987-2991.

4 W. N. Zhang, G. Lu, C. L. Cui, Y. Y. Liu, S. Z. Li, W. J. Yan, C. Xing, Y. G. Robin Chi, Y. H. Yang and F. W. Huo, Adv. Mater., 2014, 26, 4056-4060.

5 J. E. Lee, C. S. Bera, Y. S. Choi and W. I. Lee, Appl. Catal., B, 2017, 214, 15-22.

6 J. Li, Z. T. Huang, M. Yang, L. Yan, X. W. Zhang, H. Y. Gao, Y. H. Tang, Q. Q. Ma and G. Wang, New J. Chem., 2015, 39, 2949-2955.

7 J. Wang, X. L. Lu, N. Huang, H. F. Zhang, R. Z. Li and W. K. Li, Mater. Sci. Eng., B, 2017, 224, 1-8.

8 S. Bai and X. P. Shen, RSC Adv., 2012, 2, 64-98.

9 Y. W. Zhang, S. Liu, W. B. Lu, L. Wang, J. Q. Tian and X. P. Sun, Catal. Sci. Technol., 2011, 1, 1142-1144.

10 T. Fujigaya, C. Kim, Y. Hamasaki and N. Nakashima, Sci. Rep., 2016, 6, 21314.

11 O. Parlak, A. P. F. Turner and A. Tiwari, J. Mater. Chem. B, 2015, 3, 7434-7439.

12 P. T. Yin, S. Shah, M. Chhowalla and K. B. Lee, Chem. Rev., 2015, 115, 2483-2531.

13 W. G. Ma, X. Y. Lv, D. X. Han, F. H. Li, X. D. Dong and L. Niu, J. Electroanal. Chem., 2013, 690, 111-116.

14 J. Lan, H. Y. Zou, Q. Wang, P. Zeng, Y. F. Li and C. Z. Huang, Talanta, 2016, 161, 482-488.

15 Y. Y. Liu, H. C. Li, B. Guo, L. J. Wei, B. Chen and Y. Y. Zhang, Biosens. Bioelectron., 2017, 91, 734-740.

16 Y. Shen, D. J. Rao, Q. L. Sheng and J. B. Zheng, Microchim. Acta, 2017, 184, 3591-3601.

17 S. M. Kim and Y. T. Jeong, Trans. Electr. Electron. Mater., 2009, 11, 85-88.

18 K. Kim, H. Ryoo, Y. M. Lee and K. S. Shin, J. Colloid Interface Sci., 2010, 342, 479-484.
19 S. Malynych, I. Luzinov and G. Chumanov, J. Phys. Chem. B, 2002, 106, 1280-1285.

20 D. X. Li, A. He, Y. Cui and J. B. Li, Chem. Mater., 2007, 19, 412-417.

21 D. X. Li, Y. J. Jang, J. U. Lee, J. E. Lee, S. T. Kochuveedu and D. H. Kim, J. Mater. Chem., 2011, 21, 16453-16460.

22 J. E. Lee, K. C. Chung, Y. H. Jang, Y. J. Jang, S. T. Kochuveedu, D. X. Li and D. H. Kim, Anal. Chem., 2012, 84, 6494-6500.

23 H. B. Wang, B. Y. Xia, Y. Yan, N. Li, J. Y. Wang and X. Wang, J. Phys. Chem. B, 2013, 117, 5606-5613.

24 T. Kavitha, I. K. Kang and S. Y. Park, Polym. Int., 2015, 64, 1660-1666.

25 D. C. Marcano, D. V. Kosynkin, J. M. Berlin, A. Sinitskii, Z. Z. Sun, A. Slesarev, L. B. Alemany, W. Lu and J. M. Tour, ACS Nano, 2010, 4, 4806-4814.

26 Y. Cho, H. Y. Seo, Y. S. Yeom, P. Kumar, A. S. Lee, K. Y. Baek and H. G. Yoon, Carbon, 2016, 105, 340-352.

27 H. Nandivada, H. Y. Chen, L. Bondarenko and J. Lahann, Angew. Chem., Int. Ed., 2006, 45, 3360-3363.

28 Y. C. Wan, H. F. Teoh, E. S. Tok and C. H. Sow, J. Appl. Phys., 2015, 117, 054304-054312.

29 F. Zhang, H. Q. Cao, D. M. Yue and Z. F. Zhou, J. Phys. Chem. C, 2013, 117, 3513-3519.

30 D. X. Li, Q. He, Y. Yang, H. Möhward and J. B. Li, Macromolecules, 2008, 41, 7254-7256.

31 T. Ma, W. S. Yang, S. M. Liu, H. J. Zhang and F. Liang, Catalysts, 2017, 7, 38-47.

32 G. H. Chang, Y. L. Luo, W. B. Lu, X. Y. Qin, A. M. Asiri, A. O. Al-Youbi and X. P. Sun, Catal. Sci. Technol., 2012, 2, 800-806.

33 H. P. Li, L. L. N. Han, J. J. Cooper-White and H. Kim, Nanoscale, 2012, 4, 1355-1361.

34 Y. J. Song, J. H. Lü, B. X. Liu and C. L. Lü, $R S C A d v$., 2016, 6, 64937-64945.

35 J. S. Fang, Y. W. Zhang, Y. M. Zhou, C. Zhang, S. Zhao, H. X. Zhang and X. L. Sheng, Appl. Surf. Sci., 2017, 392, 3645. 